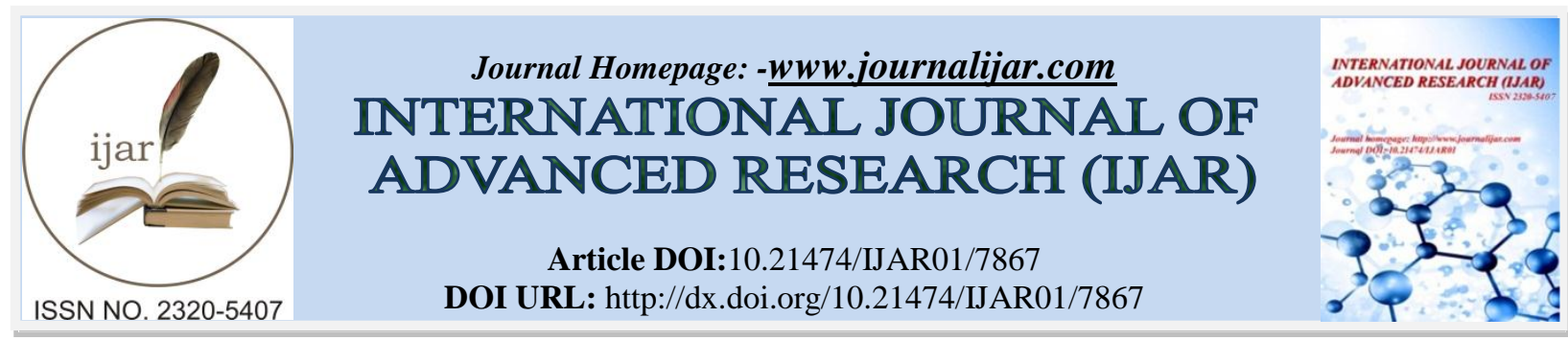

RESEARCH ARTICLE

\title{
UPDATE ON COLONOSCOPY PREPARATION, PREMEDICATION AND SEDATION.
}

\author{
Dr. Touhidul Iaslam ${ }^{1}$ and Dr. Taisir Shahriar ${ }^{2}$. \\ 1. MBBS, FCPS, MS, Consultant Depapartment of Colorecrectal Surgery, BSMMU, Shagbag, Dhaka. \\ 2. MBBS, MD (Gastroenterology).Consultant, Department Of Gastroenterology,Sono Hospital Limited,Kushtia, \\ Bangladesh.
}

\section{Manuscript Info}

Manuscript History

Received: 9 August 2018

Final Accepted: 11 September 2018

Published: October 2018

\section{Keywords:-}

Bowel Preparation ,Timing of Preparation, Inadequate Bowel Preparation, Endoscopic Sedation, The Propofol Controversy, Computerassisted PropofolDelivery,Fospropofol Disodium ,CO2 Insufflation ,Waterassisted Colonoscopy.

\section{Abstract}

The advent of optical colonoscopy has enabled gastroenterologists to visualize the colonic mucosa. This procedure has since become the cornerstone of colon cancer screening programs. Clinicians and scientists have made great strides to fine-tune the technical aspects of this procedure and have also made important advances that allow for a more effective and safer colonoscopy. This article focuses on current research and expert opinion regarding colonoscopy preparation, premedication and sedation.

\section{Introduction:-}

Colonoscopy quality has improved during the past decade due to advances in image technology, bowel preparation and sedation techniques. Nonetheless, opportunities for further improvement in outcome measures of colonoscopy remain. For example, studies from diverse settings have shown that colonoscopy is less effective in preventing proximal compared with distal cancers. Improved detection of proximal adenomas and serrated lesions would likely help to bridge this difference in outcome between proximal and distal cancers. This article presents a state-of-the-art review of bowel preparation, premedication and sedation and describes recent studies and how these findings may one day further enhance our practice of colonoscopy.

\section{Bowel Preparation}

The ideal bowel preparation for colonoscopy safely clears the colon of fecal material, exposing the mucosa and rendering it amenable to thorough inspection. Substantial evidence exists that better colon preparation is associated with increased detection of colon polyps. Recently, several articles have reviewed the subject of bowel preparation for colonoscopy and compared the efficacy, safety and tolerability of the available formulations. Additionally, a multisociety consensus document on this subject was released by the American Society of Gastrointestinal Endoscopy (ASGE) and American College of Gastroenterology.[1] This section focuses on current research regarding dietary considerations, new preparation formulations, the timing of preparation administration and predictors that help to identify patients who are most likely to have an inadequate bowel preparation.

\section{Dietary Modification}

For decades, dietary restriction of fiber and other solid food was assumed to be necessary to achieve high-quality colon cleansing. This belief, premised upon anecdotal experience, was evaluated by Wu et al., who assessed the quality of bowel cleansing in 800 Taiwanese patients following a 4-1 polyethylene glycol (4-1 PEG) 
preparation. ${ }^{[2]}$ Patient compliance with dietary restriction and the quality of bowel cleansing were assessed, the latter using the Ottawa Bowel Preparation Scale (Ottawa), which is a validated instrument for assessing bowel preparation (Table 1). ${ }^{[3]}$ They found an inverse relationship between dietary fiber intake and the bowel cleanliness score $(\mathrm{r}=-$ 0.475; $\mathrm{p}<0.001$ ), indicating that a low-fiber diet makes for a better bowel preparation. Questions remain about diet and bowel preparation, such as whether the quality of bowel cleansing with a low-residue diet is comparable to that achieved with a liquid diet. The authors are certain that there will be more work in this field during the next few years.

\section{New Formulations}

The holy grail of colonoscopy is a bowel preparation that is both effective and well tolerated by patients. A recent meta-analysis by Belsey et al. suggested that sodium phosphate and PEG 3350-based preparations are equally effective, and both were superior to magnesium sulfate and stimulant laxative-based regimens.[4] Unfortunately, safety concerns related to the development of phosphate nephropathy have limited the role of sodium phosphate as a bowel cleansing agent for colonoscopy.

A variety of PEG-based bowel preparations are now used in the USA, including 2- and 4-1 formulations. The commercially available preparations are all osmotically balanced due to the addition of electrolytes and one formulation also contains sodium ascorbate and ascorbic acid to improve taste and purgation. Sodium sulfate solution (SuPrep $®$, Braintree Laboratories, MA, USA) is a recent entry into the purgative market and sodium picosulfate, the most widely used bowel preparation for colonoscopy in Canada and the UK, is expected to be approved in the USA in the near future. Studies designed to compare these new agents with current formulations have recently been published and are worth reviewing. Readers should be mindful, however, that these studies involve relatively small numbers of subjects and some have been designed and/or funded by industry. Caution is advisable, therefore, when interpreting these studies.

\section{PEG-3350 Powder}

PEG-3350 powder (MiraLAX®, MSD Consumer Care, NJ, USA), combined with an electrolyte drink, has become a popular alternative for precolonoscopy bowel cleansing due to its improved tolerability and lower cost when compared with many of the products that are commercially available. Two studies now indicate that PEG-3350 powder is less effective than standard 4-1 PEG preparation. In one study, researchers at Oregon Health and Science University (OR, USA) randomized 190 subjects undergoing colonoscopy to split-dose preparations of either 4-1 PEG or 238 g of PEG-3350 powder mixed with 2-1 of Gatorade® (Gatorade, IL, USA) plus four bisacodyl tablets (5 mg each).[5] A good or excellent quality bowel cleansing was achieved in 83 and 68\% of patients receiving 4-1 PEG and PEG-3350 powder, respectively $(\mathrm{p}=0.018)$. In a post-hoc analysis of their data, they found that the adenoma detection rate (ADR) was numerically higher in the 41 PEG group compared with 21 PEG-3350 powder (26.2 vs $16.1 \%$, respectively; $\mathrm{p}=0.09$ ), although the difference did not achieve statistical significance. No difference in patient tolerability for the two bowel preparations was observed.

The second study randomized 403 subjects to one of four treatment groups: 4-1 PEG, 2-1 PEG-3350 powder with Gatorade, 2-1 PEG-3350 powder plus bisacodyl (10 mg) or 2-1 PEG-3350 powder with lubiprostone (24 $\mu$ g). Four liter PEG preparation was more effective at bowel cleansing (average Ottawa score: 5.1) than the three PEG-3350 powder formulations (average Ottawa score: 6.9, 6.8 and 6.3, respectively, $\mathrm{p}<0.001$ ). Patients given PEG-3350 powder tolerated the preparation better than those receiving 4-1 PEG $(\mathrm{p}<0.001)$.[6]

\section{Sodium Picosulfate}

Sodium picosulfate is a stimulant laxative that has gained popularity in Canada, Europe and Australia as a bowel cleansing agent for colonoscopy. Marketed as Pico-Salax ${ }^{\circledR}$ (Ferring, Inc., Ontario, Canada), it combines picosulfate, magnesium oxide and citric acid to create a low-volume bowel cleansing agent. At least two clinical trials with PicoSalax have been completed in the USA, and it is currently under review by the US FDA.

The relative efficacy and tolerability of picosulfate, compared with other purgatives, were demonstrated in two pivotal studies that were designed and conducted by Canadian investigators. The first study compared picosulfate alone, picosulfate plus bisacodyl $(10 \mathrm{mg})$, and oral sodium phosphate solution in 313 patients randomized in equal numbers to each arm.[7] The dosing regimen for all three agents was standardized at 5 and 10 PM the evening before colonoscopy. Overall, colon cleansing was comparable in all three treatment arms (5.1, 5.0 and 5.1, respectively; $\mathrm{p}=0.96$ ), although the quality of cleansing within the proximal colon was significantly better with picosulfate plus bisacodyl compared with picosulfate alone and sodium phosphate. Picosulfate, both alone and 
combined with bisacodyl, was well tolerated by more patients than sodium phosphate solution $(78.9,75$ and $47.5 \%$, respectively; $\mathrm{p}<0.0001)$.

The same investigators then examined the effect of a split-dose regimen on cleansing efficacy and patient tolerability.[8] Two hundred and thirty six patients were randomized to picosulfate taken either at 5 and 10 PM the evening prior to colonoscopy or at 7 PM the evening before and again $4 \mathrm{~h}$ prior to the colonoscopy. Overall, bowel cleansing and right colon-cleansing scores were improved in the split-dose group compared with the evening prior group (4.05 and 1.22 vs 5.51 and 2.14 , respectively; $p<0.001$ for both), although tolerance for the two dosing schedules was comparable $(\mathrm{p}=0.70)$. Studies comparing picosulfate with $2-1$ and $4-1$ PEG as well as with sodium sulfate solution are needed.

\section{Methylene Blue-MMX® Tablets (Cosmo Technologies Ltd., Dublin, Ireland)}

An enormous amount of research effort is currently under way designed to enhance traditional white-light colonoscopy and improve visualization and characterization of abnormalities within the colon. Chromoendoscopy, narrow-band imaging, auto-fluoresce imaging and confocal laser endomicroscopy represent some examples of this effort. These new methods of imaging, as yet unproven to improve the quality of colonoscopy, have not been widely adopted by practitioners due to the additional time and/or cost of the equipment required. Consequently, the search for a 'red flag' technology that enhances lesion detection simply and without additional equipment or expense continues.

Repici et al. studied the safety and tolerability of an orally administered chromoendoscopic agent delivered to the large bowel using multimatrix (MMX) technology.[9] Twenty two subjects received either 200 or $400 \mathrm{mg}$ of methylene blue-MMX tablets 30 min after a standard 21 PEG preparation. Tablets utilizing MMX technology have an outer, $\mathrm{pH}$-resistant film that disintegrates when the terminal ileum is reached, resulting in the delivery of product to the distal small bowel and colon. The dye was detectable in blood 3 and $5 \mathrm{~h}$ after administration of the 200- and 400-mg tablets, respectively. Peak blood concentration was reached in a median of $16 \mathrm{~h}$. Overall, $36 \%$ of subjects experienced one or more related adverse events, although none were considered serious. Clinical studies are planned to assess staining of the colonic mucosa and the detection of colon lesions.

\section{Timing of Preparation}

Reducing the 'runway time' between the last ingestion of bowel preparation agent and colonoscopy improves visualization of the bowel, especially the proximal colon, and enhances the detection of polyps.[10,11] A metaanalysis of five randomized controlled trials performed between 2005 and 2010 involving PEG-based preparations found that split dosing resulted in improved colon cleansing and patient satisfaction when compared with a preparation taken the day prior to examination.[12] Several studies published during the past year further support this view and have even examined same-day preparation.

A study by Seo et al. examined the influence of time between last dose of PEG ingestion and start of colonoscopy on bowel preparation quality in 366 subjects.[13] Subjects consumed a low-residue diet for 3 days; 2-1 doses of PEG were administered the evening prior to the colonoscopy and another 2-1 PEG $2-8 \mathrm{~h}$ prior to colonoscopy. The primary outcome was bowel cleanliness using the Ottawa Scale. The best preparation scores, both overall and within each segment, were observed in subjects who consumed their second dose of preparation 3-5 h prior to colonoscopy (Figure 1). Additionally, the study found that the volume of PEG ingested and patient compliance with instructions also correlated with the preparation outcome. While the limitations of this observational single-center study are evident, it serves to reinforce the concepts that split dosing and patient education play important roles in maximizing the quality of bowel cleansing and consequently the quality of colonoscopy.

$$
* \mathrm{p}<0.05 \text {. }
$$

Similar findings were reported by another group of Korean investigators who prospectively studied the quality of bowel preparation in 300 subjects undergoing colonoscopy.[14] All subjects were prepared using a 4-1 PEG solution. Subjects were requested to record when they began and completed the preparation as well as the procedure start time. They found that a better quality bowel preparation was present in subjects who began their preparation less than $7 \mathrm{~h}$, and completed their preparation less than $4 \mathrm{~h}$, prior to colonoscopy. The authors conclude that the timing of the bowel preparation relative to the time of examination is more important in determining the quality of bowel preparation than the time of examination. The authors conclude that the ideal 'window of opportunity' for achieving 
the optimal bowel preparation was 4-7 h prior to start of the examination. Comparable studies in western patients are eagerly awaited.

Same-day bowel cleansing for colonoscopy has been practiced in parts of Asia, particularly Japan, for many years. Until recently, this practice was inconceivable to endoscopists in the western world. The trend toward split-dosing has changed this attitude, however, and we are now seeing an increased number of reports describing same-day preparation. Researchers from Mayo Clinic Scottsdale (AZ, USA) retrospectively reviewed the colonoscopy records of 1345 subjects who underwent afternoon colonoscopy.[15] All subjects were instructed to consume a low-fiber diet for 2 days prior to colonoscopy and were given the option of consuming a PEG-based preparation using one of the following dosing schemas: 41 PEG the evening before, 41 PEG the morning of, 21 PEG plus bisacodyl (20 mg) the morning of or 21 PEG taken the evening before and repeated the morning of colonoscopy. The primary outcome was bowel preparation quality according to the Aronchick score. Multivariate analysis indicated that preparations completed on the same day were more likely to result in a good or excellent preparation compared with the 41 evening regimen (odds ratio: 7.03; 95\% CI: 2.57-19.22; $\mathrm{p}<0.001$ ). The secondary outcomes of colonoscopy completion rates and ADR were similar for these three preparations. They concluded that a same-day PEG bowel preparation yields significantly improved colonic preparation for afternoon colonoscopy compared with preparations completed the evening prior to examination.

A study by Longcroft-Wheaten and Bhandari in the UK aimed to compare the efficacy and tolerability of sodium picosulfate in 227 subjects undergoing afternoon colonoscopy who received either two doses the day before and one dose at 8 AM the morning of colonoscopy or two doses on the day of examination (at 7 and 10 AM).[16] The group that received the same-day preparation had better bowel cleansing than the split-dose group with average and excellent preparations observed in 98.4 and $89.5 \%$, respectively $(\mathrm{p}=0.0046)$. Furthermore, patient tolerance was superior with same-day preparation compared with the split-dose regimen. ADRs were similar in both groups.

The optimal regimen for optimizing bowel cleansing, regardless of the purgative agent selected, requires that at least part of the dose be administered within 4-7 $\mathrm{h}$ of the examination. This method of split-dosing preparation maximizes bowel cleansing, especially within the proximal colon where flat adenomas and subtle serrated lesions are easily missed if the bowel wall is not well cleansed. It is time for all endoscopists, regardless of what agent(s) they prefer for purgation, to adopt a split-dose regimen for colonoscopy preparation.

\section{Inadequate Bowel Preparation}

An adequate bowel preparation is defined as one that permits the detection of all polyps $>5 \mathrm{~mm}$ in size. While providing a conceptual framework for understanding an acceptable preparation, this definition has limited utility in clinical practice. From an operational standpoint, a more useful definition of an adequate preparation is one that exposes $90 \%$ or more of the mucosal surface. Most bowel preparation rating scales further stratify the term 'adequate' into good or excellent, and 'inadequate' into fair or poor. The preparation scales differ somewhat in their definitions of each level.

Roughly one in four colonoscopies has an inadequate preparation. Prolonged examination times as well as reduced rates of cecal intubation and adenoma detection have been documented in procedures where the bowel preparation is considered to be incomplete. Furthermore, patients with inadequate cleansing are often brought back for repeat examination sooner than would otherwise be recommended. Consequently, inadequate bowel preparation limits the efficacy of colonoscopy and leads to additional costs, risk of complications and, in some instances, a lower compliance rate with screening/surveillance guidelines due to frustration and disappointment with the process.

The impact of an inadequate bowel preparation on missed lesions was recently analyzed by investigators in New York (NY, USA) and St Louis (MO, USA), who retrospectively analyzed the findings of a second colonoscopy performed in selected patients with an inadequate bowel preparation 1-3 years after the index examination.[17,18] The per-patient rates of missed adenomas were 25 (NY) and 33\% (MO). Even more impressive were the peradenoma miss rates of 42 and $48 \%$, respectively. In view of these high rates of missed lesions, it would seem that the most prudent advice for patients with poor preparation and limited visibility on examination is to interrupt the procedure and to repeat the examination within $24 \mathrm{~h}$ following additional efforts at bowel cleansing. 


\section{Predictors of Inadequate Preparation}

Older age, constipation, higher BMI and significant comorbid disease have consistently been shown to be independent predictors of patients who are more likely to have an inadequate bowel preparation.[19] An Italian multicenter study prospectively evaluated 2811 consecutive subjects undergoing colonoscopy.[20] Bowel preparation quality was rated as excellent, good, fair or poor. Based upon their multivariate analysis, they developed a clinically-based model having both a sensitivity and specificity of roughly $60 \%$. In other words, the model identified nearly two of every three patients with an inadequate bowel preparation, while misclassifying $40 \%$ of patients. As expected, male gender, older age and higher BMI were independent predictors, as were advanced diabetes, liver disease, previous colorectal surgery and Parkinson's disease. While efforts to develop a clinical predictor of inadequate bowel cleansing are worthwhile, the proposed model is unlikely to receive widespread interest until its predictive score approaches $80-90 \%$.

Along similar lines, a retrospective study by Ben-Horin et al. serves to remind us that patients with a failed bowel preparation need a more intensive regimen the second time around.[21] In their series of 6990 colonoscopies, 372 procedures (5.3\%) were considered failures due to an inadequate bowel preparation and a repeat examination was advised. Of those subjects undergoing a second examination, nearly one in four $(23 \%)$ had a failed preparation the second time around. Patients having their repeated procedure the day after their failed examination were more likely to have adequate cleansing on repeat colonoscopy compared with those having their repeat examination at a later time. Providers should recognize those patient-related factors that increase the likelihood of a suboptimal bowel preparation and modify the bowel cleansing regimen in those patients accordingly.

\section{Avoiding Inadequate Preparation}

Preventing an inadequate bowel cleansing begins with patient screening, as described in the previous section. In an open-access system, this occurs during the scheduling process, when patients should be queried about chronic constipation, laxative use or a history of poor bowel cleansing during a previous colonoscopy. The identification of individuals at highest risk of having an inadequate bowel preparation provides an opportunity for timely intervention to avoid problems during colonoscopy.

Patient education is an underappreciated element of bowel preparation. For example, some patients make only a half-hearted effort at bowel cleansing, believing that the endoscopist can 'vacuum' retained debris from the colon. Patients should understand that high-quality colonoscopy requires a high-quality bowel preparation and that active involvement of the patient in this process is critical to the success of colonoscopy.

Spiegel et al. studied the effect of a novel educational booklet designed to address patient knowledge, attitude and beliefs related to colonoscopy preparation.[22] Two hundred and sixty four subjects undergoing colonoscopy were randomized 1:1 to either receive an instructional booklet 1 week prior to their scheduled colonoscopy or not, in addition to their usual standard of care. All patients attended a short bowel preparation class that included a 10-min video on bowel preparation and a question and answer period. Bowel-cleanliness quality was assessed using the Ottawa score. Patients receiving the booklet had significantly improved bowel preparation quality versus controls in both the intention-to-treat and per-protocol analyses (4.4 vs 5.1; $\mathrm{p}=0.03$ and 4.2 vs $5.1 ; \mathrm{p}=0.005$, respectively).

Unfortunately, there are no published data systematically comparing various bowel preparations for the difficult-toprepare patient. Anecdotally, it seems that most endoscopists recommend an extended preparation regimen for patients who are at risk for or have failed colonoscopy due to inadequate preparation. Common practices include an extended period of clear liquids (2-3 days), multiday PEG-based preparations, and the use of supplemental magnesium citrate or bisacodyl tablets 48-72 h prior to colonoscopy. It seems sensible to use 41 PEG rather than a lesser volume in such patients. Ibáñez et al. prospectively assessed 51 subjects referred for repeat colonoscopy due to inadequate preparation.[23] They designed an intensive bowel preparation regimen that consisted of a low-fiber diet for $72 \mathrm{~h}$ prior to colonoscopy, a clear liquid diet for $24 \mathrm{~h}$ immediately prior to procedure, bisacodyl tablets (10 $\mathrm{mg}$ ) the evening prior to the procedure and split-dose 31 PEG preparation. Patients were advised to consume an additional liter of PEG prior to colonoscopy if their colonic effluent was not clear upon completing this modified preparation. Only $10 \%$ of their study group had an inadequate preparation, suggesting that $48 \mathrm{~h}$ of low-residue diet, followed by a conventional bowel preparation, and topped off with an additional liter of PEG for those in whom the preparation remained incomplete, achieves remarkable success in a subgroup of difficult-to-prepare patients. While these results are promising, additional work in this area is needed. 


\section{Antithrombotic Agents in the Periendoscopic Period}

The management of antithrombotic agents in the periendoscopic period has become increasingly complex in recent years due to a proliferation of available agents and their increased use for a variety of conditions including vascular and coronary artery stents, atrial fibrillation, prosthetic valves, unstable coronary disease and cerebrovascular disease. Approximately $15 \%$ of patients presenting for elective endoscopy in the USA are using one or more antithrombotic agents. Included on this list are aspirin, NSAIDs, thienopyridines (clopidrogrel, ticlopidine and prasugrel), anticoagulants (warfarin, heparin and dabigatron) and glycoprotein IIb/IIIa-receptor inhibitors. There is widespread consensus, based upon independent guidelines from the ASGE, the British Society of Gastroenterology and the European Society of Gastrointestinal Endoscopy, that most routine endoscopic procedures can be performed without cessation of NSAIDs or aspirin.[24-26] This includes upper endoscopy (esophagogastroduodenoscopy [EGD]) and colonoscopy and/or biopsy, colonic polypectomy, endoscopic dilation, percutaneous endoscopic gastrostomy, endoscopic retrograde cholangiopancreatoscopy (ERCP) and endoscopic ultrasound with fine-needle aspiration and variceal-band ligation. By contrast, it is generally recommended that aspirin be stopped for endoscopic ultrasound with fine-needle aspiration of cystic lesions, endoscopic sphincterotomy with large-balloon papillary dilation, ampullary resection as well as both endoscopic mucosal resection and endoscopic submucosal dissection.

A decision to continue antithrombotic therapy or not in patients taking a thienopyridine derivative or anticoagulant is more complicated since both the benefits and risks of these agents in this patient group are greater. The management of such patients in the periendoscopic period involves weighing the relative risks of procedure-related bleeding versus vascular thrombosis. Once again, EGD and colonoscopy and/or biopsy are often performed without discontinuing a thienopyridine or anticoagulant while their cessation is advised for most therapeutic procedures. Thienopyridines and anticoagulants both increase the risk of postpolypectomy bleeding, especially in polyps greater than $1 \mathrm{~cm}$. For patients with a low risk of thrombosis, discontinuation of these agents before polypectomy is advisable. In individuals who are at higher risk of thrombosis, the management options include maintaining drug therapy combined with prophylactic application of an endoscopic hemostatic modality, substitution of aspirin for the antithrombotic drug, bridge therapy using heparin and delaying the endoscopic procedure. A decision to discontinue antithrombotic therapy for endoscopy should be individualized and in most instances, made in conjunction with the patient's cardiologist or treating physician. A summary of these recommendations is presented in Table $2 \&$ Table 3 .

\section{Endoscopic Sedation}

Sedation is designed to depress an individual's level of consciousness and to provide anxiolysis, amnesia and, in some instances, analgesia. The introduction of propofol (2,4-di-isopropylphenol) as an agent for procedural sedation has rapidly transformed the landscape of endoscopic sedation. Much of propofol's popularity among doctors and patients is related to its pharmacokinetic and pharmacodynamic properties, which bestow the drug with 'rapid-on, rapid-off' properties and provide patients with a 'sense of well-being', respectively. In many respects, propofol is an ideal agent for brief procedures on ambulatory patients. Today, nearly half of all endoscopists in the USA perform the majority of their procedures with propofol and that figure continues to grow annually. This section will review the sedation menu and the science, economics and politics behind each option. The 'sedation package' also includes nonpharmacological efforts designed to reduce patient discomfort during colonoscopy. In this context, it is also appropriate to discuss $\mathrm{CO} 2$ insufflation and water-immersion techniques

\section{Preprocedure Fasting}

The American Society of Anesthesiologists (ASA) guidelines for preprocedural fasting have recently been updated.[27] The new version preserves the '2-6-8 rule' for preprocedure fasting - that is, a minimum fasting period of $2 \mathrm{~h}$ is required for clear liquids, $6 \mathrm{~h}$ for a light meal and $8 \mathrm{~h}$ for full meal. The idea of a 'light meal' is something of a misnomer, since it includes toast and liquids but excludes fatty foods such as butter. These recommendations are not based upon hard scientific fact but instead are derived from studies assessing gastric residual volume following variable periods of fasting.

The fasting guidelines do not preclude the use of a split-dose or even a same-day bowel cleansing regimen, provided that the minimum 2-h period of fasting is observed. Investigators in Indianapolis (IN, USA) prospectively studied 712 subjects having EGD, either alone or combined with colonoscopy, to assess the effect of a split-dose bowel preparation on residual gastric volume.[28] They observed no difference in gastric residual volume between patients who received a split-dose regimen $(19.7 \mathrm{ml})$ and patients who completed their preparation the evening prior to colonoscopy $(20.2 \mathrm{ml})$. 


\section{The Propofol Controversy}

Sedation for endoscopy has traditionally been performed by an endoscopist along with a specially trained nurse. The preferred sedation agents included a benzodiazepine combined with an opioid analgesic. The introduction of propofol by anesthesiologists for brief endoscopic procedures has forever altered how endoscopists and patients view endoscopic sedation. During the past 15 years, propofol has become the drug of choice among many endoscopists due to its favorable pharmaceutical properties and outstanding safety profile. Controversy continues to exist, however, regarding the administration of propofol by a trained nurse working under the supervision of an endoscopist. In spite of an evidence-based consensus statement issued jointly by ASGE, American College of Gastroenterology, the American Gastroenterological Association and American Association for the Study of Liver Disease supporting the practice, the ASA continues to maintain that propofol should be administered only by anesthesia providers.[29] This debate was fueled by the Centers for Medicare and Medicaid Services interpretative guidelines on deep sedation issued in 2009-2010, which seemed to support the position that propofol administration should be limited to anesthesia specialists.[101] The practical and economic implications of their directive have been described by Rex.[30] He points out that the routine use of an anesthesia provider to oversee sedation during endoscopy could add as much as US $\$ 5$ billion annually to the USA healthcare budget. Several alternatives, including endoscopist-directed propofol, computer-assisted propofol delivery and new sedation agents with product labels that permit their use by nonanesthesiologists may one day provide acceptable, lower-cost options for procedural sedation.

While American anesthesiologists have continued to oppose endoscopist-directed propofol, their counterparts in Europe proved to be more open-minded and objective, or so it seemed. Following a comprehensive review of the literature, representatives of the European Society of Gastrointestinal Endoscopy, European Society of Gastroenterology and Endoscopy Nurses and Associates, and the European Society of Anesthesiology (ESA) released consensus guidelines that endorsed nonanesthesiologist administration of propofol and provided a framework for training.[31] Within several months of its publication, 21 national societies of anesthesiology within Europe, representing roughly half of all member societies within ESA, disassociated themselves from the European document "out of concern for patient safety" and reaffirmed the ASA's position that propofol should be administered only by those trained in the administration of general anesthesia.[32] Not long after, the remaining members of ESA followed suit, resulting in the ESA officially retracting its support of non-anesthesiologist administration of propofol.[33] Although endoscopist-directed propofol has been practiced safely and effectively in several European countries having formalized guidelines and training curricula, its future within these and other European countries is now uncertain in light of the shift in position by the anesthesia societies. Readers are encouraged to stay tuned since the last chapter of this story has yet to be written.

\section{Computer-assisted Propofol Delivery}

Imagine a device that could calculate and deliver the appropriate loading and maintenance dose of propofol, monitor vital signs and adjust the infusion rate of propofol in response to changes in physiologic parameters and the patient's level of response. Such a device, called SEDASYS ${ }^{\circledR}$ (Ethicon Endo-Surgery, OH, USA), was actually designed to provide moderate sedation using target-controlled infusion of propofol and a proprietary closed-loop system that adjusts the infusion rate of propofol. In a prospective, randomized multicenter study comparing SEDASYS® to conventional sedation using midazolam and an opioid, subjects sedated by the propofol delivery system experienced less hypoxemia than those receiving standard sedation.[34] A total of 1000 patients undergoing EGD or colonoscopy were randomized with roughly equal numbers of patients in each arm. The primary end point was oxygen desaturation area under the curve, which is the summed difference between the threshold oxygen saturation (defined as 90\%) and the subject's oxygen saturation during the examination. The investigators observed that the hypoxemia area under the curve was significantly higher (more oxygen desaturation) for those receiving standard sedation compared to the computer-assisted propofol group ( 88.8 vs $23.6 \mathrm{~s} \% ; \mathrm{p}=0.028$ ). Additionally, both patient and clinician satisfaction were significantly greater in the computer-assisted propofol group compared with standard sedation. No serious adverse events were observed in the propofol group versus three with standard sedation. The device received an 'approvable letter' from the FDA upon appeal following an initial decision of 'nonapprovable'. It will be interesting to see how this device fits in to the overall landscape of endoscopic sedation in various countries around the world.

\section{Fospropofol Disodium}

Fospropofol (LUSEDRA ${ }^{\mathrm{TM}}$ injection, Eisai, NJ, USA) is an inactive prodrug of propofol that is converted enzymatically by alkaline phosphatases to the active agent. Fospropofol was conceived based upon the idea that the 
prodrug would produce lower peak concentrations and a slightly increased time to peak plasma propofol concentration compared with the parent compound, producing an agent that would retain the pharmacodynamic benefits of propofol while producing a more predictable propofol level. The longer duration of effect also meant less need for repeated dosing during procedures of short duration. In several multicenter studies, fospropofol has proven to be an effective agent for procedural sedation. At a loading dose of $6.5 \mathrm{mg} / \mathrm{kg}$ and supplemental doses of 1.6 $\mathrm{mg} / \mathrm{kg}$ (25\% of the loading dose), fospropofol produced moderate sedation without ventilatory insufficiency.[35] Unfortunately, the future of fospropofol as a moderate sedation agent is uncertain at present due to the FDA's decision that mandated the manufacturer to include a monitored anesthesia care label, identical to propofol's label, in fospropofol's package insert. Consequently, the commercial use of fospropofol has been limited largely to anesthesiologists.

\section{CO2 Insufflation}

Gaseous distension of the colon due to air insufflation can produce significant bloating and abdominal discomfort. As much as 8-14 1 of gas, usually room air, is pumped into the bowel during colonoscopy, compared with only 11 in the physiologic state. $\mathrm{CO} 2$, a gas with properties of high diffusability and rapid absorption/excretion, has been used since the 1980 s as an alternative to air under the assumption that it produced less distension. During the past decade, well-designed, randomized, controlled trials performed in different clinical settings have defined the benefits of $\mathrm{CO} 2$ insufflation in colonoscopy as well as double-balloon enteroscopy and endoscopic retrograde cholangiopancreatography.

These studies demonstrate that patients experience less pain and discomfort after colonoscopy when the bowel is insufflated with $\mathrm{CO} 2$ compared with room air. This conclusion is supported by two recent meta-analyses.[36,37] Wu et al. analyzed nine randomized controlled trials comprising 1577 patients undergoing colonoscopy.[36] Compared with air, $\mathrm{CO} 2$ insufflation resulted in less pain during the procedure (relative risk: $0.77 ; 95 \% \mathrm{CI}: 0.62-0.96$ ) as well as 1 -h $(0.26 ; 0.16-0.43)$, 6-h $(0.36 ; 0.20-0.64)$ and $24-\mathrm{h}(0.53 ; 0.31-0.91)$ postprocedure. Viewed in another way, the use of $\mathrm{CO} 2$ insufflation reduced postprocedure discomfort in 50,33 and $8 \%$ of patients at 1,6 and $12 \mathrm{~h}$, respectively. Additionally, $\mathrm{CO} 2$ insufflation eliminates the small, but real, risk of combustion when electrocautery is used within the colon. No differences in safety or cecal intubation rate have been observed, and the doses of sedation administered are comparable with $\mathrm{CO} 2$ and air insufflation. Notably, $\mathrm{CO} 2$ insufflation has not been established to reduce patient discomfort during colonoscopy.

Suzuki et al. studied the effect of CO2 insufflation on ventilation and acid-base balance in a group of patients having colonoscopy with CO2 insufflation under general anesthesia.[38] They found that CO2 insufflation is safe during endoscopy, even in longer procedures such as endoscopic mucosal resection and endoscopic submucosal dissection. Small changes were observed in arterial pressure of $\mathrm{CO} 2$ and end-tidal $\mathrm{CO} 2$, although values remained within the physiologic range.

In a recent editorial on $\mathrm{CO} 2$ insufflation, Bretthauer questioned why only a small fraction of endoscopists worldwide routinely use CO2.[39] He identified several barriers to adoption, including cost (including the initial outlay for equipment and the per-procedure cost of $\mathrm{CO} 2$ ), lack of awareness by endoscopists and the absence of promotion by instrument and device companies. Bretthauer urged professional gastrointestinal societies to incorporate recommendations for routine $\mathrm{CO} 2$ use into future clinical practice guidelines.

\section{Water-assisted Colonoscopy}

In contrast with $\mathrm{CO} 2$ insufflation, which reduces postprocedure discomfort but has little effect on intraprocedural pain, the water-assisted method was developed to reduce procedural pain and maximize the success rate of unsedated colonoscopy. In addition, water-infused techniques facilitate advancement of the colonoscope during difficult intubations. The water-assisted technique is based upon the simple concept that infusing the left colon with water causes it to drop into the pelvis and become straightened. Additionally, warm water relaxes the bowel and minimizes spasm. As a result, scope passage through the left colon is simplified and discomfort is lessened. As described by Leung, the air pump is turned off prior to instrument insertion to avoid lengthening of the colon due to air distension of the bowel, and all residual air and stool are suctioned out.[40]

Leung et al. have performed several pivotal studies of water-assisted colonoscopy. In one trial, 227 subjects were randomized to either air or water insufflation.[41] The study's primary end point, complete colonoscopy with minimal sedation (midazolam $2 \mathrm{mg}$ ), was achieved in 51 and 28\% ( $\mathrm{p}<0.05$ ) with water and air, respectively. Rates 
for cecal intubation (100\%) and lesion detection were similar in both the groups. A recent review of published randomized trials comparing water exchange with air insufflation found that 11 of 13 studies reported a significant reduction of pain score during or after colonoscopy, and two trials described lower doses of sedation drugs or fewer patients with severe pain using water-related methods.[42]

In a study comparing ADRs, 368 subjects were randomized to water- or air-assisted colonoscopy. The ADRs were 57.1 and $46.1 \%(\mathrm{p}=0.04)$ with water and air, respectively, suggesting that lesion detection might be improved with water irrigation and aggressive suctioning of residual colonic contents.[43]

\section{Conclusions:-}

This article summarizes recent developments in colonoscopy with particular emphasis on three areas: bowel preparation, premedication and endoscopic sedation. Three important concepts warrant special consideration. First, split-dose bowel preparation remains a key concept for enhancing the quality of colonoscopy, especially the proximal colon. This observation comes at a time when the value of colonoscopy within the proximal colon is being debated. Some authors have even opined that the timing of preparation administration is more important than the formula itself. Endoscopists around the world should embrace the principle of split-dose preparation. Second, endoscopists are encouraged to become familiar with the new antithrombin and antiplatelet drugs that are being used increasingly by patients who present for elective endoscopy. Based upon the specific procedure being performed, a decision analysis is required to decide whether to maintain the agents during the periprocedural period and accept a risk of bleeding or to discontinue such drugs prior to the examination and expose the patient to an increased risk of thrombosis and its sequelae. Third, endoscopists everywhere continue to struggle over what is appropriate sedation for endoscopy. Has propofol become the standard of care, as some endoscopists believe, or is its use 'discretionary' as others have indicated?[44] These and other related issues make endoscopic sedation possibly the most contentious topic within the field of gastroenterology today.

\section{Acknowledgements:-}

We would like to thanks Dr. A.K.M. Munir, MBBS, Ph. D and Dr. Tahmina Begum, MBBS, Dr.Sadia Afrin MBBS, FCPS (MEDICINE) part -1, MRCP (UK), part -1 for their valuable advice. We alsoacknowledge Mr. Shah AbulAwal for his computer assistance.

\section{Expert Commentary}

Colonoscopy is widely considered to be the gold standard for the detection of colorectal polyps and cancer. Nevertheless, there is an opportunity for improvement in our current methods of practice. For example, the miss rates for colorectal polyps are reported to be up to $24 \%$ for all lesions, and up to $11 \%$ for advanced adenomas. The recent emphasis on high quality bowel preparation with particular focus on the proximal colon has been demonstrated to improve the detection rates of all colon neoplasms, especially flat lesions within the right side of the colon that may be difficult to visualize. Greater awareness of these hard to detect lesions, along with enhanced attention to the quality of bowel preparation, have been shown to increase polyp detection and reduce the number of missed lesions.

\section{Five-year View}

Quality is a cornerstone of the new healthcare landscape. As endoscopists, we will be held accountable not merely for completing our procedures but also for their quality and appropriateness. Evidence-based quality measures, designed to assess the outcomes of our procedures, are being developed and will be judged accordingly. The ADR, a validated measure of quality that correlates closely with the risk of an interval carcinoma after colonoscopy, will probably remain the most important outcome measure of screening colonoscopy.

Other quality measures, such as the number of adenomas per patient and number of sessile serrated lesions per patient, are being assessed as well. Methods to improve lesion detection, including better quality bowel preparation, cap-assisted colonoscopy, water-assisted colonoscopy and image-enhanced techniques such as chromoendoscopy and autofluorescence imaging are all under active investigation.

The 'quality imperative' is also focused on the efficient utilization of resources. Better understanding of an individual's colorectal cancer risk, based upon their family and personal history will permit better stratification of 
patients into different surveillance intervals. We are likely to see better guidance in the management of patients with one or more sessile serrated lesions within the proximal colon.

Finally, the primacy of colonoscopy as the gold standard for colorectal cancer screening will be challenged during the next 5 years. Fecal immunochemical testing and stool DNA both pose real threats to colonoscopy, based upon their simplicity and cost. As endoscopists, we must heed the quality initiative and ensure that each of us provide high quality examinations to the right patients at the right times.

\section{References:-}

1. Chak A. Endoscopic ultrasonography. Endoscopy 2000; 32: 146-152

2. Giovannini M, Monges G, Seitz JF, et al. Distant lymph node metastases in esophageal cancer: impact of endoscopic ultrasound-guided biopsy. Endoscopy 1999; 31: 536-540

3. Wallace MB, Hawes RH, Sahai AV, et al. Dilation of malignant esophageal stenosis to allow EUS guided fine-needle aspiration: safety and effect on patient management. GastrointestEndosc 2000; 51: 309-313

4. Van Dam J, Rice TW, Catalano MF, et al. High-grade malignant stricture is predictive of esophageal tumor stage: risks of endosonographic evaluation. Cancer 1993; 71: 2910-2917

5. Catalano MF, Van Dam J, Sivak MV Jr. Malignant esophageal strictures: staging accuracy of endoscopic ultrasonography. GastrointestEndosc 1995; 41: 535-539

6. International Journal of Advancements in Research \& Technology, Volume 5, Issue 6, June-2016 96

7. ISSN 2278-7763

8. Salminen JT, Farkkila MA, Ramo OJ, et al. Endoscopic ultrasonography in the preoperative staging of adenocarcinoma of the distal oesophagus and oesophagogastric junction. Scand J Gastroenterol 1999; 34: 1178-1182

9. Schlick T, Heintz A, Junginger T. The examiner's learning effect and its influence on the quality of endoscopic ultrasonography in carcinoma of the esophagus and gastric cardia. SurgEndosc 1999; 13: 894-898

10. Wallace MB, Hoffman BJ, Sahai AS, et al. imaging of esophageal tumors with a water-filled condom and a catheter US probe. GastrointestEndosc 2000; 51: 597-600

11. Aabakken L, Silvestri GA, Hawes R, et al. Cost-efficacy of endoscopic ultrasonography with fine-needle aspiration vs. mediastinotomy in patients with lung cancer and suspected mediastinaladenopathy. Endoscopy 1999; 31: 707-711

12. Mishra G, Sahai AV, Penman ID, et al. Endoscopic ultrasonography with fine-needle aspiration: an accurate and simple diagnostic modality for sarcoidosis. Endoscopy 1999; 31: 377-382

13. Ohashi S, Segawa K, Okamura S, et al. The utility of endoscopic ultrasonography and endoscopy in the endoscopic mucosal resection of early gastric cancer. Gut 1999; 45: 599-604

14. Kojima T, Takahashi H, Parra-Bianco A, et al. Diagnosis of submucosal tumor of the upper GI tract by endoscopic resection. GastrointestEndosc 1999; 50: 516-522

15. Palazzo L, Landi B, Cellier C, et al. Endosonographic features predictive of benign and malignant gastrointestinal stromal cell tumours. Gut 2000; 46: 88-92

16. Gress FG, Hawes RH, Savides TJ, et al. Role of EUS in the preoperative staging of pancreatic cancer: a large single center experience. GastrointestEndosc 1999; 50: 786-791

17. Erickson RA, Sayage-Rabie L, Beissner S. Factors predicting the number of EUS-guided fine-needle passes for diagnosis of pancreatic malignancies. GastrointestEndosc2000 ; 51: 184-190

18. Voss M, Hammel P, Molas G, et al. Value of endoscopic ultrasound guided fine needle aspiration biopsy in the diagnosis of solid pancreatic masses. Gut 2000; 46: 244-249

19. Gress F, Gottlieb K, Cummings O, et al. Endoscopic ultrasound characteristics of mucinous cystic neoplasms of the pancreas. Am J Gastroenterol 2000; 95: 961-965

20. Ardengh JC, Rosenbaum P, Ganc AJ, et al. Role of EUS in the preoperative localization of insulinomas compared with spiral CT. GastrointestEndosc 2000; 51: 552-555

21. Kubo H, Chijiiwa Y, Akahoshi K, et al. Pre-operative staging of ampullarytumours by endoscopic ultrasound. Br J Radiol 1999; 72: 443-447

22. Cannon ME, Carpenter SL, Elta GH, et al. EUS compared with CT, magnetic resonance imaging, and angiography and the influence of biliary stenting on staging accuracy of ampullary neoplasms. GastrointestEndosc 1999; 50: 2733

23. Hastier P, Buckley MJM, Francois R, et al. A prospective study of pancreatic disease in patients with alcoholic cirrhosis: comparative diagnostic value of ERCP and EUS and long-term significance of isolated parenchymal abnormalities. GastrointestEndosc 1999; 49: 705-709

24. Sahai AV, Mishra G, Penman ID, et al. EUS to detect evidence of pancreatic disease in patients with persistent or nonspecific dyspepsia. GastrointestEndosc 2000; 52: 153-159

25. Tamada K, Wada S, Ohashi A, et al. Intraductal US in assessing the effects of radiation therapy and prediction of patency of metallic stents in extrahepatic bile duct carcinoma. GastrointestEndosc 2000; 51: 405-411 
26. Chak A, Isenberg G, Kobayashi K, et al. Prospective evaluation of an over-the-wire catheter US probe. GastrointestEndosc 2000; 51: 202-205

27. Menzel J, Poremba C, Dietl KH, Domschke W. Preoperative diagnosis of bile duct strictures: comparison of intraductal ultrasonography with conventional endosonography. Scand J Gastroenterol 2000; 35: 77-82

28. Polkowski M, Palucki J, Regula J, et al. Helical computed tomographic cholangiography versus endosonography for suspected bile duct stones: a prospective blinded study in non-jaundiced patients. Gut 1999; 45: 744-749

29. Liu CL, Lo CM, Chan JKF, et al. EUS for detection of occult cholelithiasis in patients with idiopathic pancreatitis. GastrointestEndosc 2000; 51: 28-32

30. Lachter J, Rubin A, Shiller M, et al. Linear EUS for bile duct stones. GastrointestEndosc 2000; 51: 51-54

31. Sugiyama M, Atomi Y, Yamato T. Endoscopic ultrasonography for differential diagnosis of polypoid gall bladder lesions: analysis in surgical and follow up series. Gut 2000; 46: 250-254

32. Fujita N, Noda Y, Kobayashi G, et al. Diagnosis of the depth of invasion of gallbladder carcinoma by EUS. GastrointestEndosc 1999; 50: 659-663

33. McClave SA, Jones WF, Woolfolk GM, et al. Mistakes on EUS staging of colorectal carcinoma: error in interpretation or deception from innate pathologic features? GastrointestEndosc 2000; 51: 682-689

34. Norton SA, Thomas MG. Staging of rectosigmoidneoplasia with colonoscopicendoluminal ultrasonography. Br J Surg 1999; 86: 942-946

35. Lohnert MS, Doniec JM, Henne-Bruns D. Effectiveness of endoluminalsonography in the identification of occult local rectal cancer recurrence. Dis Colon Rectum 2000; 43: 483-491

36. Spinelli P, Schiavo M, Meroni E, et al. Results of EUS in detecting perirectal lymph node metastases of rectal cancer: the pathologist makes the difference. GastrointestEndosc 1999; 49: 754-758

37. Magdeburg B, Fried M, Meyenberger C. Endoscopic ultrasonography in the diagnosis, staging, and follow-up of anal carcinomas. Endoscopy 1999; 31: 359-364

38. Williams DB, Sahai AV, Aabakken L, et al. Endoscopic ultrasound guided fine needle aspiration biopsy: a large single centre experience. Gut 1999; 44: 720-726

39. Sahai AV, Schembre D, Stevens PD, et al. A multicenter U.S. experience with EUS-guided fine-needle aspiration using the Olympus GF-UM30P echoendoscope: safety and effectiveness. GastrointestEndosc 1999; 50: 792-796

40. Seifert H, Dietrich C, Schmitt T, et al. Endoscopic ultrasoundguided one-step transmural drainage of cystic abdominal lesions with a large-channel echo endoscope. Endoscopy $2000 ; 32: 255-259$

41. Lahoti S, Catalano MF, Alcocer E, et al. Obliteration of esophageal varices using EUS-guided sclerotherapy with color Doppler. GastrointestEndosc 2000; 51: 331-333

42. Lee YT, Chan FKL, $\mathrm{Ng} \mathrm{EKW,} \mathrm{et} \mathrm{al.} \mathrm{EUS-guided} \mathrm{injection} \mathrm{of} \mathrm{cyanoacrylate} \mathrm{for} \mathrm{bleeding} \mathrm{gastric} \mathrm{varices.}$ GastrointestEndosc 2000; 52: 168-174

43. Nguyen P, Feng JC, Chang KJ. Endoscopic ultrasound ( EUS ) and EUS-guided fine-needle aspiration (FNA) of liver lesions. GastrointestEndosc 1999; 50: 357-361

44. Erickson RA, Tretjak Z. Clinical utility of endoscopic ultrasound and endoscopic ultrasound-guided fine needle aspiration in retroperitoneal neoplasms. Am J Gastroenterol 2000; 95: 1188-1194

45. Goldberg SN, Mallery S, Gazelle GS, Brugge WR. EUS-guided radiofrequency ablation in the pancreas: results in a porcine model. GastrointestEndosc 1999; 50: 392-401

46. Chak A, Isenberg G, Mallery S, et al. Prospective comparative evaluation of video US endoscope. GastrointestEndosc1999; 49: 695-699

47. Nomura N, Goto H, Niwa Y, et al. Usefulness of contrast-enhanced EUS in the diagnosis of upper GI tract disease. GastrointestEndosc 1999; 50: 555-560

48. Tamada K, Tomiyama T, Ohashi A, et al. Preoperative assessment of extrahepatic bile duct carcinoma using threedimensional intraductal US. GastrointestEndosc 1999; 50: 548-554

49. Allescher HD, Rösch T, Willkomm G, et al. Performance, patient acceptance, appropriateness of indications and potential influence on outcome of EUS: a prospective study in 397 consecutive patients. GastrointestEndosc 1999; 50: 737-745

50. Nickl N. Endosonography at a crossroads: the outcomes obligation. GastrointestEndosc 1999; 50: 875-878

51. Kim LS, Koch J. Do we practice what we preach? Clinical decision making and utilization of endoscopic ultrasound for staging esophageal cancer. Am J Gastroenterol 1999; 94: 1847- 1852 